\title{
Methotrexate nanoparticle delivery system for treatment of inflammatory bowel disease in pediatric patients
}

\author{
Gang Liu, Dun-Chen Li, Ping-Ping Li, Ran-Ran Li and Shu-Ying Chen* \\ Department of Paediatrics, Affiliated Hospital of Taishan Medical University, Shandong 271000, China
}

*For correspondence: Email: chenshuyingsy@hotmail.com; Tel/Fax: 0086-0538-6236376

Received: 20 February 2016

Revised accepted: 14 June 2016

\begin{abstract}
Purpose: To evaluate the efficacy and safety of methotrexate (MTX) nanoparticles in pediatric patients with inflammatory bowel disease (IBD).

Methods: In this randomized, open-label clinical study, 28 pediatric patients with moderate to severe IBD were randomly assigned to treatment (MTX nanoparticles, $15 \mathrm{mg} /$ week) or control (azathioprine, $A Z A, 2 \mathrm{mg} / \mathrm{kg} /$ day) group. Nanoparticles were synthesized by adding calcium chloride to sodium alginate solution containing MTX, and was further treated with poly-L-lysine aqueous solution. The nanoparticles were evaluated for particle size, zeta potential and drug encapsulation efficacy. Erythrocyte sedimentation rate, C-reactive protein, aspartate aminotransferase, alanine transaminase, and disease activity scores were used to assess IBD remission.

Results: Nanoparticle size, zeta potential and encapsulation efficacy were $164.4 \pm 6.9 \mathrm{~nm},-32.6 \pm 3.7$ $m V$, and $97.8 \pm 4.2 \%$, respectively. After 12 weeks of therapy, the mean Pediatric Crohn's Disease Activity Index (PCDAl) scores for control and treatment groups were $22.3 \pm 2.14$ and $16.8 \pm 1.87$, respectively, while mean Pediatric Ulcerative Colitis Activity (PUCAI) Index scores were $24.3 \pm 1.47$ and $18.7 \pm 1.92$, respectively. Eight patients in the treatment and five patients in the control group achieved remission. Biochemical parameters varied significantly between the groups.

Conclusion: MTX nanoparticles are safe and more effective than standard first-line IBD therapy. However, further studies are required to determine the suitability of the formulation for therapeutic use.
\end{abstract}

Keywords: Pediatric patient, Methotrexate nanoparticle, Inflammatory bowel disease, Azathioprine

\begin{abstract}
Tropical Journal of Pharmaceutical Research is indexed by Science Citation Index (SciSearch), Scopus, International Pharmaceutical Abstract, Chemical Abstracts, Embase, Index Copernicus, EBSCO, African Index Medicus, JournalSeek, Journal Citation Reports/Science Edition, Directory of Open Access Journals (DOAJ), African Journal Online, Bioline International, Open-J-Gate and Pharmacy Abstracts
\end{abstract}

\section{INTRODUCTION}

Inflammatory bowel disease (IBD) is a multifactorial disorder characterized by an inappropriate immune response that includes Crohn's disease (CD) and ulcerative colitis (UC), which are chronic inflammatory intestinal idiopathic conditions [1]. IBD may present at any age; however, UC and CD occur most frequently during the second and third decades of life, particularly during adolescence [2,3]. The incidence of IBD, CD in particular, has increase in recent years [1].
MTX has been shown to effectively induce and maintain remission of $C D$ in adult and pediatric patients [2,3], but the effect of MTX on refractory IBD was first reported by Kozarek et al [4]. Five controlled and several uncontrolled studies have demonstrated the effectiveness of MTX in inducing remission of steroid-dependent or chronic active CD [5-7] and as a steroid-sparing agent for the treatment of refractory IBD. Furthermore, decreasing the use of steroids may reduce the incidence of steroid-related adverse effects in patients with chronic IBD. High-dose 
MTX is associated with several side effects including nausea, vomiting, headache, abdominal pain, diarrhea, and skin rash.

Nanoparticles are designed to control drug release after oral administration, which may reduce the dosage frequency as well as the incidence of adverse events. Furthermore, nanoparticles may protect encapsulated drugs from mucosal metabolism, thus increasing intracellular drug concentrations [8].

Alginic acid and sodium and potassium alginates are widely used mucoadhesive biomaterials because of their good biocompatibility, biodegradation [9], sol-gel transition properties, and chemical versatility, which allow for further modifications [10]. From a regulatory perspective, the United States Food and Drug Administration (USFDA) considers alginates to be "generally recognized as safe" (GRAS), a designation that applies to substances accepted as safe for alimentary use [11]. GRAS excipients are listed in the Code of Federal Regulations Title 21 parts 182 and 184 [12]. Thus, because of its good biocompatibility, suitability for oral use, and USFDA approval, alginate is extensively used in the development of nanomedicines.

We evaluated the safety and efficacy of MTXalginate nanoparticles in pediatric patients with IBD.

\section{EXPERIMENTAL}

\section{Materials}

MTX was obtained from BBI (Boston, MA, USA). Sodium alginate with a molecular weight of 12,000-40,000 and poly-L-lysine were purchased from Sigma-Aldrich (Steinheim, Germany). Calcium chloride $\left(\mathrm{CaCl}_{2}\right)$ and sodium chloride $(\mathrm{NaCl})$ were purchased from Merck Chemicals [Darmstadt, Germany]. All reagents were of analytical grade.

\section{Patient selection and study design}

We conducted a randomized, open-label, clinical pilot study at Taishan Medical University Hospital. The study protocol was approved by the local ethics committee of Taishan Medical University (approval no. 201367143). Children and adolescents between the ages of 8 and 17 years with moderate to severe IBD were recruited. Patients with a Pediatric Ulcerative Colitis Activity Index (PUCAI) score $>34$ or a Pediatric Crohn's Disease Activity Index (PCDAI) score $>30$ were included in the study. Exclusion criteria included ileostomy or colostomy, toxic megacolon, prior sensitivity or allergy to MTX, and having received anti-tumor necrosis factor- $\alpha$ (TNF- $\alpha$ ) in the previous 8 weeks. Written informed consent was obtained from all parents, and verbal consent was obtained from all participants. The study was conducted according to the International Conference on Harmonization (ICH) Good Clinical Practices (GCP) and in compliance with the Declaration of Helsinki 1975 $[13,14]$.

All eligible patients were randomly assigned to the treatment (MTX nanoparticles equivalent to $15 \mathrm{mg} /$ week for 12 weeks) or control (AZA, 2 $\mathrm{mg} / \mathrm{kg} /$ day orally for 12 weeks) group. AZA was chosen as the comparison treatment because it has been widely used as a first-line therapy for IBD and is therefore a good standard for this test of the efficacy of MTX nanoparticles. All patients in the treatment group received a daily folic acid supplement. Corticosteroids were used by 64.2 $\%$ of patients in the treatment group and by 71.4 $\%$ in the control group. The steroid dose was decreased by $5 \mathrm{mg} /$ week in patients whose condition was stable at week 4 . All patients were restricted from taking immunosuppressants, antibiotics, and aminosalicylates during the study.

\section{MTX nanoparticle preparation}

MTX nanoparticles were synthesized using the method reported by Rajaonarivony et al [15]. Briefly, $4 \mathrm{~mL}$ of $18 \mathrm{mM} \mathrm{CaCl}_{2}$ was added to 76 $\mathrm{mL}$ of $0.06 \%$ sodium alginate solution containing MTX while stirring. Then, $16 \mathrm{~mL}$ of $0.05 \% \mathrm{w} / \mathrm{v}$ of a poly-L-lysine aqueous solution was added. The solution was stirred for $2 \mathrm{~h}$ and kept at room temperature overnight. Subsequently, the solution was centrifuged at $18,000 \mathrm{rpm}$ for 30 min and washed three times with distilled water to acquire the nanoparticles.

\section{Nanoparticle characterization}

\section{Particle size}

The mean particle size was determined using photon correlation spectroscopy (Nanophox system, Sympatec $\mathrm{GmbH}$, Clausthal-Zellerfeld, Germany). The MTX-loaded nanoparticle formulation was diluted with deionized water to a pertinent scattering intensity. The data analysis was performed using the cumulative method for spherical particles. The results were evaluated as the effective diameter, and the polydispersity index (PDI) was calculated as the relative width of the particle size distribution. 


\section{Zeta potential}

The zeta potential of the MTX nanoparticle formulation was determined using a Nanosight NS500 (Malvern Instruments, Ltd., Malvern, UK). To obtain the zeta potential, the MTX nanoparticle sample was diluted in doubledistilled water and placed in an electrophoretic cell.

\section{Fourier transform infrared spectroscopy (FTIR)}

The FTIR transmission spectra were determined using a FTIR-8400S spectrophotometer (Shimadzu, Kyoto, Japan). The sample for analysis was prepared by mixing pure drug and nanoparticle samples with potassium bromide powder ( $1 \%$ of the potassium bromide amount). A potassium bromide disc was prepared using hydraulic compression at 10,000 psi. The potassium bromide disc was scanned over wavelength regions of $400-4000 \mathrm{~cm}^{-1}$.

\section{Drug encapsulation efficiency}

The drug encapsulation efficiency of the nanoparticle formulation was obtained by separation of MTX nanoparticles from the aqueous medium holding free MTX. The formulation was first placed into the dialysis bag (Spectra/Por® 3,500 MWCO, Spectrum Medical Industries, Los Angeles, CA, USA), which allowed dialysis of the free drug until the encapsulated drug was complete released. During dialysis, the medium, $0.9 \% \mathrm{NaCl}$, was changed every $30 \mathrm{~min}$, to monitor the amount of free drug. The formulation was considered separated when free drug could no longer be detected. Triton $X 100(0.1 \mathrm{~mL})$ was added to 2 $\mathrm{mL}$ of the sample solution, which produced a clear solution. The encapsulation efficiency $(E \%)$ of the MTX nanoparticles was measured by ultraviolet spectrophotometer (UV-2600, Shimadzu) at $303 \mathrm{~nm}$ and calculated as in Equation 1,

$E(\%)=([D a-D b] / D a) 100$

where $\mathrm{Da}$ and $\mathrm{Db}$ are the drug concentrations after and before dialysis, respectively.

\section{In vitro release studies}

In vitro drug release from the MTX nanoparticles formulation was achieved by dialysis in a phosphate-buffered saline solution at $\mathrm{pH}$ 7.4. The optimized MTX nanoparticles (equivalent to $2.5 \mathrm{mg}$ of the drug) were placed in a dialysis bag and kept in $50 \mathrm{~mL}$ of dissolute on a medium; they were then placed in shaker incubator that was persistently rotated at $100 \mathrm{rpm}$ at $37^{\circ} \mathrm{C}$.

A small amount of solution was withdrawn for the analysis. The amount of MTX released from the nanoparticles was determined spectrophotometrically at $303 \mathrm{~nm}$. Each experimental procedure was performed three times. The cumulative release of MTX at each time point was calculated as the ratio of drug released at any time ( $t$ ) to the initial amount of encapsulated drug; that value is expressed as a percentage.

\section{Measurement of therapeutic efficacy}

Our primary outcome was the feasibility and safety of administering MTX nanoparticles to children as assessed by the number and severity of adverse reactions. The secondary outcome was the efficacy of MTX nanoparticles on disease activity. A positive clinical response was defined as a 10-point decrease from baseline in the PCDAl or PUCAI score, with scores $<10$ considered to indicate inactive disease or complete remission.

Patients were withdrawn from the study in cases of severe infection, persistent thrombocytopenia or leukopenia, imminent surgery, unacceptable adverse reaction, deterioration of disease symptoms, noncompliance, or on the patient's request.

\section{Patients follow up}

Patients were followed up at 2, 4, 8, and 12 weeks during treatment and every 4 weeks for 6 consecutive months after the trial. At each followup visit, the PCDAl and PUCAI scores were evaluated and the patients underwent a complete blood count, hemoglobin, C-reactive protein (CRP), aspartate aminotransferase (AST), alanine transaminase (ALT), and erythrocyte sedimentation rate (ESR) tests.

\section{Statistical analysis}

All pre- and post-treatment data were assessed using Student's paired t-test. All data are expressed as means \pm standard error (SE). $P$ values $<0.05$ were deemed to indicate statistical significance. All categorical variables were assessed using the Chi-square test. Adverse events were reported quantitatively and qualitatively using the Common Terminology Criteria for Adverse Events (CTCAE). The Statistical Package for the Social Sciences version 9 (SPSS, Inc., Chicago, IL, USA) was used to conduct the statistical tests. 


\section{RESULTS}

\section{Patient clinical characteristics}

The baseline characteristics of patients in each study arm are shown in Table 1 . Of 33 patients screened for the study, 5 were excluded because of prior history of colostomy $(n=1)$, exposure to infliximab $(n=1)$ and mild disease $(n=3)$. Thus, 28 patients (17 males and 11 females) were enrolled in the study, and 14 patients were randomly assigned to each arm of the study. A total of 21 patients had CD and 7 had UC. The mean age of participants at the time of enrolment was 13.15 years. Corticosteroids were used by $64.2 \%$ of the patients in the treatment group and by $71.4 \%$ in the control group.

\section{MTX nanoparticle characteristics}

Table 2: MTX nanoparticle characteristics

\begin{tabular}{lc}
\hline Characteristic & $\begin{array}{l}\text { Value (mean } \pm \\
\text { SEM) }\end{array}$ \\
\hline Entrapment efficiency $(\%)$ & $97.8 \pm 4.2$ \\
Particle size $(\mathrm{nm})$ & $164.4 \pm 6.9$ \\
Zeta potential $(\mathrm{mV})$ & $-32.6 \pm 3.7$ \\
\hline
\end{tabular}

The MTX nanoparticle characteristics are shown in Table 2. Particle size is the most important characteristic of the nanoparticle formulation. The mean particle size was $164.4 \mathrm{~nm}$, with PDI of 0.218 . The low PDI indicated a uniform particle size distribution. The encapsulation efficacy was $97.8 \%$. The zeta potential was $-32.6 \mathrm{mV}$, indicating good stability of the formulation, which was attributable to a decrease in electrostatic repulsion between particles, which were stabilized by the formation of a coat over their surfaces.

\section{FTIR spectra}

The FTIR spectra of MTX and the MTX-alginate nanoparticles are shown in Fig. 1. Significant peaks were observed at $3391 \mathrm{~cm}^{-1}$ due to $-\mathrm{NH}$ stretching as well as the characteristic MTX peaks at 1644 and $1603 \mathrm{~cm}^{-1}$. In the spectrum of the MTX-alginate nanoparticle formulation, significant peaks were observed at 3437, 1644, and $1603 \mathrm{~cm}^{-1}$. Additional peaks were present due to the presence of polymer, indicating no interactions between the polymer and drug in this nanoparticle formulation.

\section{In-vitro drug release}

Fig. 2 shows the in vitro drug release of the MTX nanoparticles. The MTX nanoparticles showed an initial burst release of approximately $32.7 \%$, followed by a sustained release for up to $48 \mathrm{~h}$. The early burst release may have been the result of drug precipitation from the nanoparticles or drug absorbed over the nanoparticles surface. The slow diffusion of the drug from the polymer matrix resulted in prolonged release.

\section{Treatment response}

The study included patients with moderate to severe disease. At baseline, the mean PCDAl and PUCAI scores were 48 (range, 33-59) and 49.1 (range, 35-61), respectively. No significant differences were observed between groups. However, after 4, 8, and 12 weeks of treatment, the PCDAI (Fig. 3) and PUCAI (Fig. 4) scores were significantly different between groups.

Table 1: Patient baseline characteristics according to group

\begin{tabular}{lll}
\hline Characteristic & Control group & Treatment group \\
\hline No. of patients & 14 & 14 \\
Male (female) & $9(5)$ & $8(6)$ \\
Age, (years), mean \pm SEM (Range, & $12.7 \pm 1.6(9.1-16.8)$ & $13.6 \pm 1.4(8.6-16)$ \\
years) & & \\
BMl (range), & $19.6 \pm 1.5(15.4-25.6)$ & $18.2 \pm 1.2(14.4-$ \\
$\mathrm{kg} / \mathrm{m}^{2}$ & & $25.2)$ \\
Type of disease & & \\
CD & 10 & 11 \\
UC & 4 & 3 \\
PCDAl & $48.6 \pm 2.2$ & $47.3 \pm 1.9$ \\
PUCAI & $49.1 \pm 2.3$ & $49.2 \pm 1.7$ \\
AST & $37.4 \pm 2.9$ & $39.3 \pm 2.3$ \\
ALT & $19.3 \pm 1.7$ & $15.7 \pm 1.3$ \\
CRP & $3.8 \pm 2.1$ & $4.1 \pm 1.7$ \\
ESR & $38.4 \pm 2.3$ & $41.3 \pm 1.9$ \\
\hline
\end{tabular}

$\mathrm{BMI}=$ Body Mass Index; $\mathrm{CD}=$ Crohn's Disease; UC = Ulcerative Colitis; PCDAI = Paediatric Crohn's Disease Activity Index; PUCAI = Paediatric Ulcerative Colitis Activity Index; AST = Aspartate transaminase; ALT = Alanine transaminase; CRP = C-reactive protein; ESR = erythrocyte sedimentation rate] 


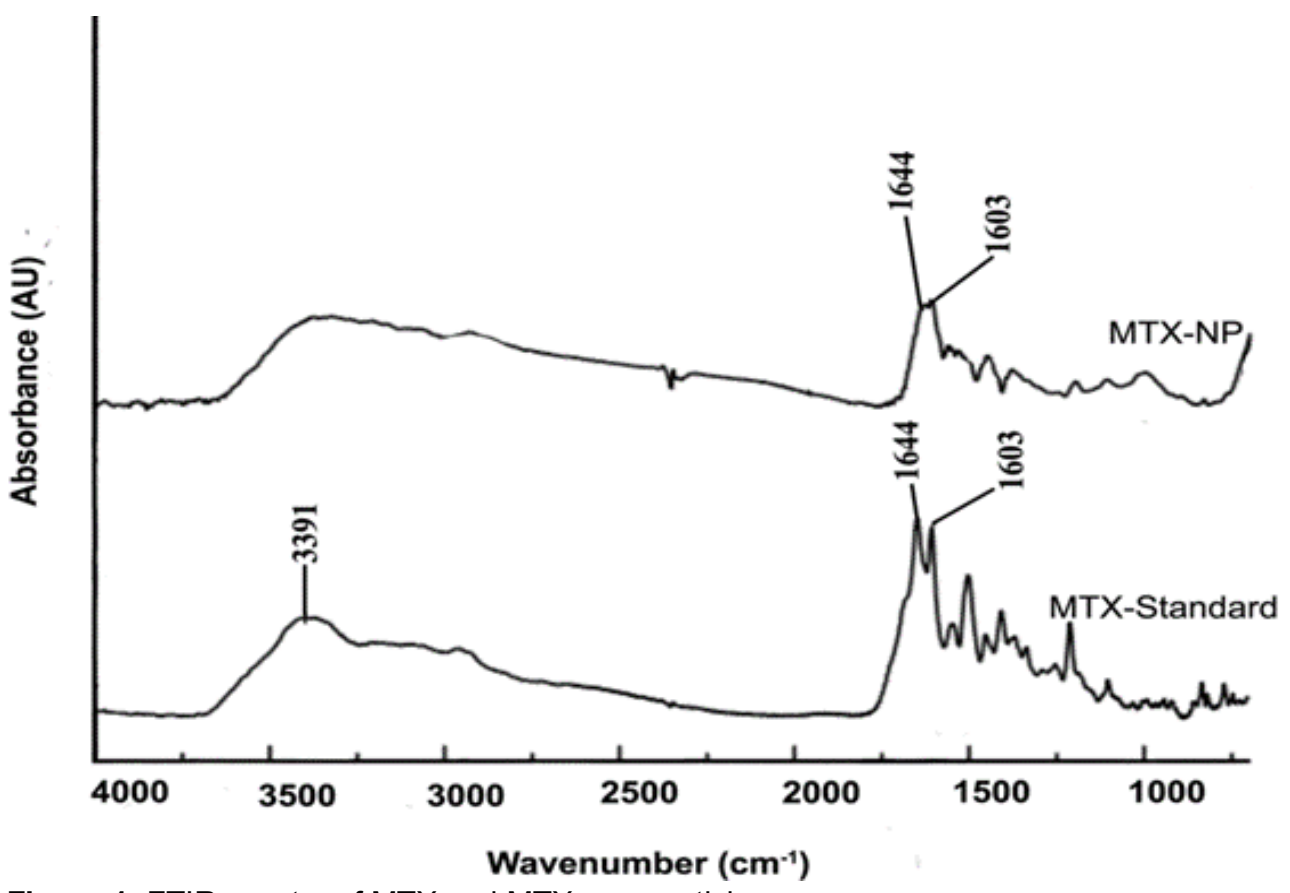

Figure 1: FTIR spectra of MTX and MTX nanoparticles

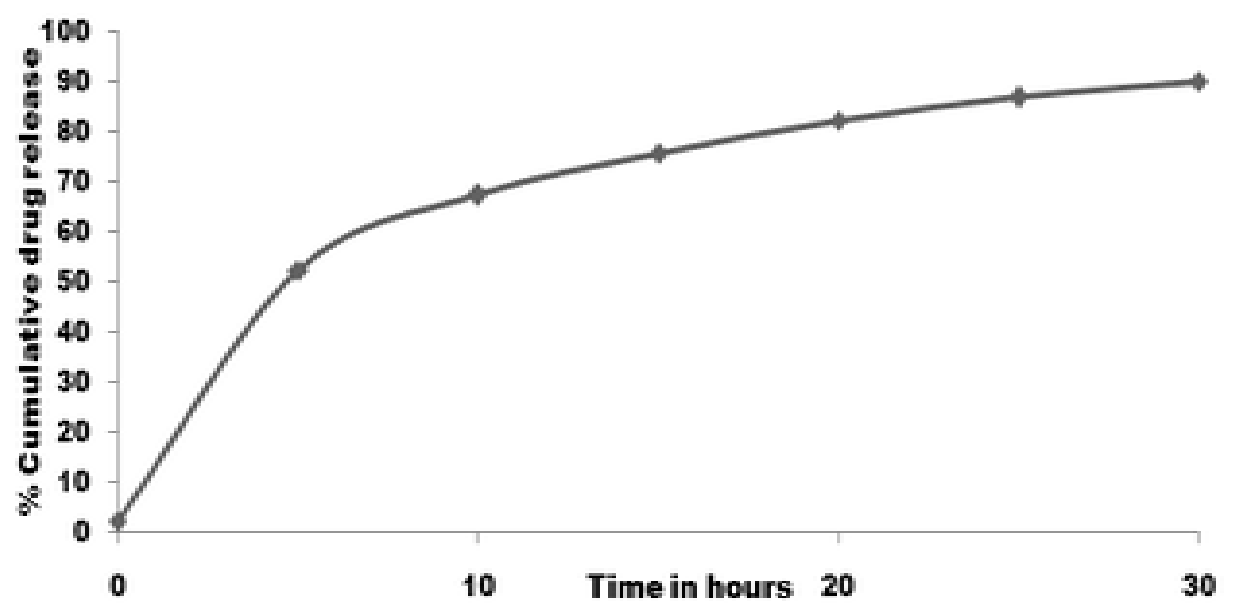

Figure 2: Release profile of the MTX nanoparticles

At 12 weeks, the mean PCDAl scores for the control and treatment groups were $22.3 \pm 2.14$ and $16.8 \pm 1.87$, respectively $(p=0.022)$. Similarly, the mean PUCAI scores for the control and treatment groups were $24.3 \pm 1.47$ and 18.7 \pm 1.92 , respectively $(p=0.022)$. Table 3 shows the laboratory findings for the control and treatment groups according to treatment duration. The mean CRP and ESR values were significantly different between groups at 8 and 12 weeks. At the beginning of the study, 9 patients in the treatment group and 10 in the control group were using steroids. However, the steroid dose decreased significantly in both groups, and 12 patients ( 8 in MTX group and 5 in AZA group) were steroid free by the end of the study. After 12 weeks of treatment, 13 of 28 patients achieved remission, 8 in the treatment group and 5 in the control group.

\section{Toxicity}

The adverse events observed during the study are shown in Table 4. No deaths were reported in either group. The commonly reported adverse events were thrombocytopenia, leukopenia, nausea, and vomiting in the AZA group and thrombocytopenia, leukopenia, and liver enzyme elevation in the MTX group. No serious adverse events were reported in either group, with the exception of one patient in the AZA group, who developed grade 3 leukopenia, which resolved after discontinuation of the drug. No patients in the treatment group required discontinuation of the treatment. 
Table 3: Laboratory findings in the treatment and control groups according to treatment duration

\begin{tabular}{|c|c|c|c|c|c|c|c|c|c|}
\hline \multirow[b]{2}{*}{ Test } & \multicolumn{3}{|c|}{ Week 4} & \multicolumn{3}{|c|}{ Week 8} & \multicolumn{3}{|c|}{ Week 12} \\
\hline & Control & $\begin{array}{c}\text { Treatme } \\
n t\end{array}$ & P-value & $\begin{array}{c}\text { Contro } \\
I\end{array}$ & $\begin{array}{c}\text { Treatmen } \\
t\end{array}$ & $\begin{array}{c}P \\
\text { value }\end{array}$ & Control & $\begin{array}{c}\text { Treatme } \\
n t\end{array}$ & $\begin{array}{c}P- \\
\text { value }\end{array}$ \\
\hline $\begin{array}{l}\text { CRP } \\
\text { (mg/L) }\end{array}$ & $\begin{array}{c}2.9 \pm \\
0.11\end{array}$ & $\begin{array}{r}2.5 \pm \\
0.12\end{array}$ & $0.031^{*}$ & $\begin{array}{c}2.4 \pm \\
0.21\end{array}$ & $\begin{array}{c}2.01 \pm \\
0.17\end{array}$ & $0.013^{*}$ & $\begin{array}{l}1.8 \pm \\
0.19\end{array}$ & $\begin{array}{l}1.2 \pm \\
0.23\end{array}$ & $0.023^{*}$ \\
\hline $\begin{array}{l}\text { ESR } \\
(\mathrm{mm} / \mathrm{h})\end{array}$ & $\begin{array}{c}34.4 \pm \\
1.53\end{array}$ & $\begin{array}{c}32.6 \pm \\
1.72\end{array}$ & 0.35 & $\begin{array}{c}26.6 \pm \\
1.93\end{array}$ & $\begin{array}{c}21.4 \pm \\
1.36\end{array}$ & $0.012^{*}$ & $\begin{array}{c}19.7 \pm \\
2.13\end{array}$ & $\begin{array}{c}13.9 . \pm \\
1.87\end{array}$ & $0.0413^{*}$ \\
\hline $\begin{array}{l}\text { AST } \\
(U / L)\end{array}$ & $\begin{array}{c}33.9 \pm \\
2.37\end{array}$ & $\begin{array}{c}31.6 \pm \\
1.97\end{array}$ & 0.40 & $\begin{array}{c}31.4 \pm \\
2.17\end{array}$ & $\begin{array}{c}28.9 \pm \\
2.76\end{array}$ & 0.36 & $\begin{array}{c}29.7 \pm \\
1.85\end{array}$ & $\begin{array}{c}24.3 \pm \\
2.13\end{array}$ & $0.035^{*}$ \\
\hline $\begin{array}{l}\mathrm{ALT} \\
(\mathrm{U} / \mathrm{L})\end{array}$ & $\begin{array}{c}16.3 \pm \\
1.47\end{array}$ & $\begin{array}{c}20.6 \pm \\
1.54\end{array}$ & $0.02^{*}$ & $\begin{array}{c}19.7 \pm \\
1.77\end{array}$ & $\begin{array}{c}21.9 \pm \\
1.95\end{array}$ & 0.49 & $\begin{array}{c}20.6 \pm \\
1.69\end{array}$ & $\begin{array}{c}24.3 \pm \\
1.37\end{array}$ & 0.1 \\
\hline
\end{tabular}

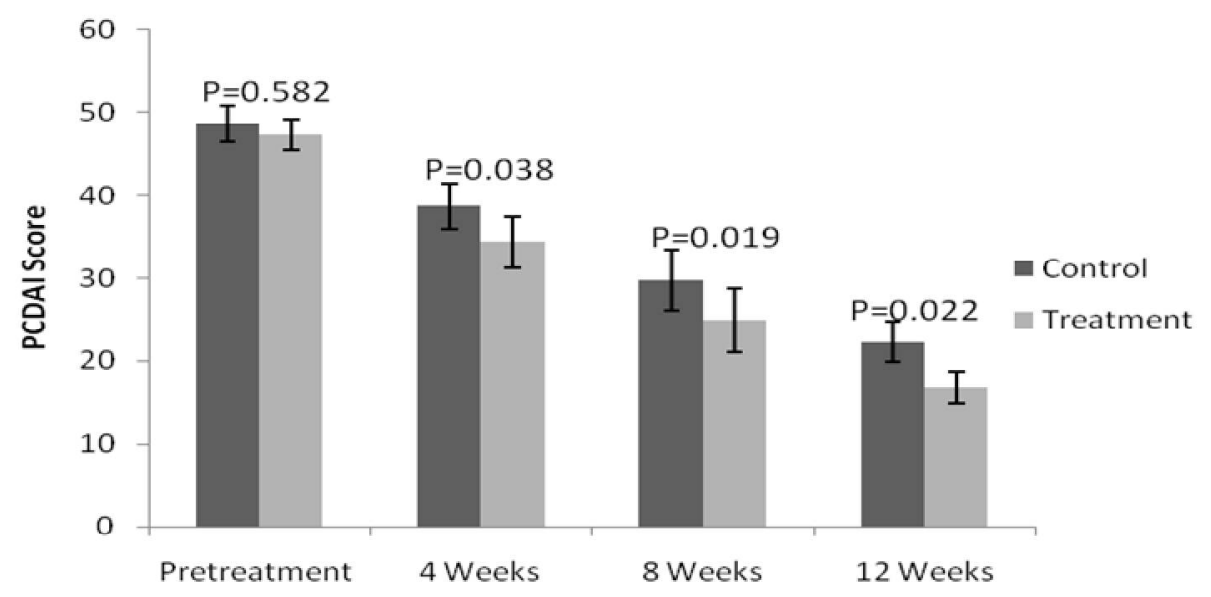

Figure 3: PCDAI Score of each group at different time intervals of study

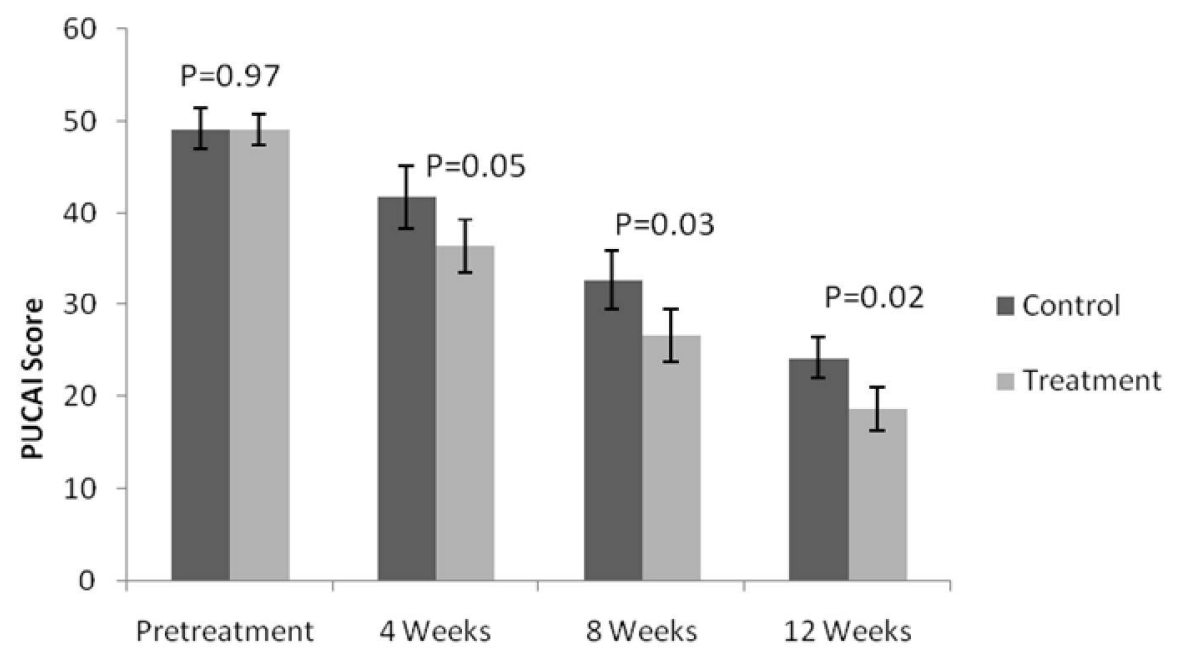

Figure 4: PUCAI Score of each group at different time intervals of study

Table 4: Adverse events reported in the study

\begin{tabular}{lcc}
\hline Adverse events & Control group & Treatment group \\
\hline Thrombocytopenia & 4 & 1 \\
Leukopenia & $2^{*}$ & 1 \\
Nausea & 4 & 0 \\
Vomiting & 5 & 0 \\
Elevated lever enzyme & 0 & 2 \\
\hline${ }^{*}$ One patient developed grade 3 leukopenia & &
\end{tabular}




\section{DISCUSSION}

Although several recent studies have investigated the effect of nanoparticle formulations in cell culture and small-animal models, particularly rodents, few have evaluated the effect of nanoparticle formulations in humans. Schmidt et al [16] were the first to use nanoparticles to treat patients with IBD. We evaluated the safety and efficacy of MTX nanoparticles administered to children with moderate to severe active IBD. We compared the performance of MTX nanoparticles against that of AZA. The baseline disease activity scores (PCDAI and PUCAI), ESR, CRP, and monthly steroid dose were similar between groups $(p>$ 0.05).

A previous study found that a low dose of oral MTX administered weekly was a safe and effective steroid-sparing agent for patients with CD who did not respond to other therapies [8]. Based on this finding, we used a low dose of MTX in our study. Several controlled studies have investigated the effect of MTX in patients with IBD. In a double-blind, randomized, placebocontrolled study of MTX in chronic active UC, Oren et al [17] found no advantage of MTX over placebo in patients with moderate to severe UC. By contrast, we found that the PUCAI scores following treatment with MTX nanoparticles were significantly lower than those in the control group.

Ardizzone et al [18] found that MTX was effective in inducing remission in patients with chronic active CD; however, its efficacy was comparable to that of AZA [18]. By contrast, we found that MTX nanoparticles were significantly more effective than AZA. The greater effectiveness of the MTX nanoparticle may be due to the properties of nanoparticles. Several previous studies have shown that nanoparticle formulations have increased therapeutic efficacy and low toxicity compared with standard treatments, such as rolipram, 5-ASA, tacrolimus, and heparin [19]. Furthermore, nanoparticles help encapsulated drugs evade P-glycoprotein, the mucosal enzyme that metabolizes drugs, resulting in increased intracellular concentration of the drugs [20].

We found that the disease activity scores were significantly lower in the treatment than in the control group. A significant number of patients in both groups achieved remission; however, more patients in the MTX group achieved complete remission. Thus, our findings clearly indicate that MTX nanoparticles provide significant advantages over conventional AZA therapy. Moreover, we observed fewer and less severe adverse events than reported in previous MTX trials, and it was not necessary to discontinue treatment because of toxicity.

Our favorable results may be due to the targeted and sustained drug delivery provided by the nanoparticle formulation. Schmidt et al. [16] reported that nanoparticles accumulated in the ulcerated lesions of patients with IBD. The increased efficacy and low toxicity observed in our study may be due to a similar accumulation effect. Moreover, several animal and cell culture studies have demonstrated preferential uptake of nanoparticles into inflamed tissue [[21]. Thus, it may be that the comparatively high efficacy and low toxicity of the MTX nanoparticle treatment observed in our study resulted from the capacity of the nanoparticles to shield the drug from systemic absorption and to be preferentially taken up and accumulate in the inflamed tissue.

\section{Limitations of the study}

The findings of this study was limited by the small sample size and the open-label nature of the design, which may be subject to bias. The random assignment of patients to the treatment and control groups reduced the likelihood of bias; however, further randomized double-blinded clinical trials are needed to confirm our results.

\section{CONCLUSION}

Several studies have shown that MTX is a good alternative in patients who cannot tolerate or are resistant to AZA/6MP treatment. Due to the high cost of immunosuppressant drugs, MTX should be considered before prior to drug therapy. The occurrence of systemic adverse effects is the major limitation of MTX therapy; however, this limitation can be overcome by using a nanoparticle drug delivery system. The selective targeting of inflamed tissue underlies the increased efficacy and low toxicity of nanoparticle formulations compared with conventional therapy. Thus, MTX nanoparticles would be safer and more effective than the standard first-line treatment in pediatric patients.

\section{DECLARATIONS}

\section{Acknowledgement}

This study was supported by Taishan Medical University Hospital, Shandong, China. 


\section{Conflict of Interest}

No conflict of interest associated with this work.

\section{Contribution of Authors}

The authors declare that this work was done by the authors named in this article and all liabilities pertaining to claims relating to the content of this article will be borne by them.

\section{REFERENCES}

1. Molodecky NA, Soon IS, Rabi DM, Ghali WA, Ferris M, Chernoff G, Benchimol El, Panaccione R, Ghosh S, Barkema HW, Kaplan GG. Increasing incidence and prevalence of the inflammatory bowel diseases with time, based on systematic review. Gastroenterology 2012; 142(1): 46า-54.

2. Feagan $B G$, Fedorak RN, Irvine EJ, Wild G, Sutherland L, Steinhart AH, Greenberg GR, Koval J, Wong CJ, Hopkins $M$, et al. A comparison of methotrexate with placebo for the maintenance of remission in Crohn's disease. N Engl J Med. 2000; 342: 1627-1632.

3. Uhlen S, Bellbouab R, Narebski K, Goulet O, Schmitz J, Cézard JP, Turck D, Ruemmele FM. Efficacy of methotrexate in pediatric Crohn's disease: a French multicenter study. Inflamm Bowel Dis. 2006; 12: 10531057.

4. Cummings JR, Herrlinger KR, Travis $S P$, Gorard $D A$, Mclntyre AS, Jewell DP. Oral methotrexate in ulcerative colitis. Aliment Pharmacol Ther. 2005; 21: 385-389.

5. Wahed M, Louis-Auguste JR, Baxter LM, Limdi JK, McCartney SA, Lindsay JO, Bloom SL. Efficacy of methotrexate in Crohn's disease and ulcerative colitis patients unresponsive or intolerant to azathioprine /mercaptopurine. Aliment Pharmacol Ther. 2009; 30 : 614-620.

6. Egan LJ, Sandborn WJ, Tremaine WJ, Leighton JA, Mays DC, Pike MG, Zinsmeister AR, Lipsky JJ. A randomized dose-response and pharmacokinetic of methotrexate for refractory inflammatory Crohn's disease and ulcerative colitis. Aliment Pharmacol Ther. 1999; 13: 1597-1604.

7. Lamprecht A, Schafer U, Lehr CM. Size-dependent bioadhesion of micro- and nanoparticulate carriers to the inflamed colonic mucosa. Pharm. Res. 2001; 18: 788793.

8. Cho K, Wang X, Nie S, Chen ZG, Shin DM. Therapeutic nanoparticles for drug delivery in cancer. Clin Cancer Res. 2008; 14(5): 1310-1316.
9. Pawar SN, Edgar KJ. Alginate derivatization: a review of chemistry, properties and applications. Biomaterials 2012; 33(11): 3279-3305.

10. Swain S, Behera A, Beg S, Patra CN, Dinda SC, Sruti J, Rao ME. Modified alginate beads for mucoadhesive drug delivery system: an updated review of patents. Recent Recent Pat Drug Deliv Formul. 2012; 6(3): 259277.

11. Chang D, Chang RK. Review of current issues in pharmaceutical excipients. Pharmaceut Technol. 2007; 31(5): $56-66$.

12. Sodium Alginate, CFR - Code of Federal Regulations Title 21; 2013.

13. International Conference on Harmonization of Technical Requirements for Registration of Pharmaceuticals for Human Use (ICH) Topic E6 Note for Guidance on Good Clinical Practice: Consolidated Guideline. London: European Agency for the Evaluation of Medicinal Products 1996

14. Carlson RV, Boyd KM, Webb DJ. The revision of the Declaration of Helsinki: past, present and future. $\mathrm{Br} J$ Clin Pharmacol 2004; 57(6): 695-713.

15. Rajaonarivony $M$, Vouthier $C$, Couarrze G, Puisieux $F$, Couvreur $P$. Development of a new drug carrier made from alginate, J Pharm Sci, 1993; 82: 912-917.

16. Schmidt $C$, Lautenschlaeger $C$, Collnot EM, Schumann M, Bojarski C, Schulzke JD, Lehr CM, Stallmach A. Nano- and micro scaled particles for drug targeting to inflamed intestinal mucosaา-A first in vivo study in human patients. J Control Release. 2013; 165(2): 139145.

17. Oren $R$, Moshkowitz $M$, Odes $S$, Becker $S$, Keter $D$, Pomeranz I, Shirin H, Reisfeld I, Broide E, Lavy A et al. Methotrexate in chronic active Crohn's disease: a double-blind, randomized, Israeli multicenter trial. Am J Gastroenterol. 1997; 92: 2203-2209.

18. Ardizzone S, Bollani S, Manzionna G, Imbesi V, Colombo E, Bianchi Porro G. Comparison between methotrexate and azathioprine in the treatment of chronic active Crohn's disease: a randomized, investigator-blind study. Dig Liver Dis. 2003; 35(9): 619-627.

19. Lamprecht A, Yamamoto $H$, Takeuchi H, Kawashima Y. A pH-sensitive microsphere system for the colon delivery of tacrolimus containing nanoparticles. J Control Release 2005; 104(2): 337-346.

20. Ruperto N, Martini A. Emerging drugs to treat juvenile idiopathic arthritis. Expert Opin Emerg Drugs. 2011; 16: 493

21. Lamprecht A, Yamamoto $H$, Takeuchi H, Kawashima $Y$. A $\mathrm{pH}$-sensitive microsphere system for the colon delivery of tacrolimus containing nanoparticles. $J$ Control Release. 2005; 104(2): 337-346. 